Pacific

Journal of

Mathematics

\title{
TUNNEL ONE, FIBERED LINKS
}

MATt RATHBUN

Volume 259 No. 2

October 2012 


\title{
TUNNEL ONE, FIBERED LINKS
}

\author{
MATt RATHBUN
}

\begin{abstract}
For a fibered link of tunnel number one in $S^{3}$, with fiber $F$ and unknotting tunnel $\tau$, we show that $\tau$ can be isotoped to lie in $F$.
\end{abstract}

\section{Introduction and motivation}

The study of fibered knots and links is as important today as ever. Giroux's correspondence [2002] between open book decompositions and contact structures mingles classical fibered links with more modern contact geometry. Sutured manifold theory continues to reveal information about fibrations (see, for instance, [Ni 2009; Scharlemann and Thompson 2009]). And fibered links are related to the newest advances in Floer homology, as knot Floer homology detects fibered links [Ni 2007] and sutured Floer homology intersects both contact geometry and sutured manifold theory.

Tunnel number one links are among the most studied links. Much of the work on tunnel number one links revolves around trying to isotope the tunnel to sit nicely with respect to some additional structure in the 3-manifold, including a hyperbolic metric [Adams 1995; Adams and Reid 1996; Akiyoshi et al. 1997; Cooper et al. 2010], polyhedral decompositions [Sakuma and Weeks 1995; Heath and Song 2005], bridge decompositions [Goda et al. 2000; Lackenby 2005], Seifert surfaces [Scharlemann and Thompson 2003], and fibrations [Sakuma 1996]. These studies, and others, have led to the classification of tunnels for many classes of knots and links, including torus knots [Boileau et al. 1988], satellite knots [Morimoto and Sakuma 1991], nonsimple links [Eudave Muñoz and Uchida 1996], 2-bridge knots [Morimoto and Sakuma 1991; Kobayashi 1999], and 2-bridge links [Morimoto 1994; Jones 1995].

Further, the Berge conjecture states that if a knot admits a lens space Dehn surgery, then it is in one of the families of knots classified by John Berge. Many are working on this long-standing conjecture, with recent progress contributed by Ozsváth and Szabó [2005], Hedden [2007], Baker, Grigsby, and Hedden [2008],

The author would like to thank Abigail Thompson. The author was supported in part by NSF VIGRE Grants DMS-0135345 and DMS-0636297, and the RTG Grant DMS-0739208.

MSC2010: primary 57M25; secondary 57M27.

Keywords: tunnel, fibered, knot, link, monodromy. 
Saito [2007], and Williams [2007], among others. Yi Ni [2007] recently proved that if a knot admits a lens space surgery, then it is a fibered knot. Additionally, all Berge knots are both fibered and tunnel number one, so further understanding of tunnel one, fibered knots could have profound impacts on the conjecture.

Jesse Johnson [2008] investigated genus-2 Heegaard splittings of closed surface bundles over the circle. This paper looks at the relationship between unknotting tunnels and fibrations for link complements.

Theorem 1.1. Let $K$ be an oriented, fibered, tunnel number one link in $S^{3}$, with fiber $F$, and unknotting tunnel $\tau$. Then $\tau$ can be isotoped to lie in $F$.

\section{Background and definitions}

\section{3-manifolds.}

Notation 1. Let $A$ be subset of a 3-manifold $M$. We fix some notation. Let $n(A)$ denote a small open neighborhood of $A$ in $M$. If $F$ is a properly embedded surface in $\mathrm{M}$, let $M \mid F=M \backslash n(F)$. If $S$ is the boundary of $M$, we will refer to $S \mid \partial F=$ $S \backslash n(\partial F)$. For convenience, we will also sometimes refer to this as $S \mid F$.

Definition 2.1. Let $F$ be a surface properly embedded in a 3-manifold $M$. Then $F$ is said to be compressible if there exists a disk $D$ embedded in $M$ with $\partial D=D \cap F$ an essential curve in $F$, and $D$ is called a compressing disk for $F$. If $F$ is not compressible, and is not a 2-sphere, then it is called incompressible. The surface $F$ is said to be boundary compressible if there exists a disk $D$ embedded in $M$ with $D \cap F=\alpha \subset \partial D, D \cap \partial M=\beta \subset \partial D$, where $\alpha$ is an essential arc in $F$, $\alpha \cap \beta=\partial \alpha=\partial \beta$, and $\alpha \cup \beta=\partial D$. In this case, $D$ is called a boundary compression disk. If $F$ is not boundary compressible, it is called boundary incompressible.

Definition 2.2. A compression body $V$ is the result of taking the product of a surface with [0,1], attaching 2-handles along $S \times\{0\}$, and then attaching 3-handles along any resulting 2-sphere components. The surface $S \times\{1\}$ is called $\partial_{+} V$, and $\partial V \backslash \partial_{+} V$ is called $\partial_{-} V$. A handlebody is a compression body where $\partial_{-} V=\varnothing$. A Heegaard splitting is a triple $(S, V, W)$, where $S$ is a surface, $V$ and $W$ are compression bodies, $\partial_{+} V=\partial_{+} W=S$, and $M=V \cup_{S} W$.

Definition 2.3. Let $K$ be a knot in a 3-manifold $M$, and let $\lambda$ be an essential closed curve in $\partial n(K)$. Let $M^{\prime}$ be the manifold obtained from $M$ by removing $n(K)$, and attaching a solid torus $S^{1} \times D^{2}$ to $M \backslash n(K)$ via a homeomorphism of the boundaries such that $\{\mathrm{pt}$. $\} \times \partial D^{2}$ is identified with the curve $\lambda$. Then $M^{\prime}$ is said to be the result of $\lambda$-sloped Dehn surgery on $M$. 


\section{Tunnels.}

Definition 2.4. A link $L$ in $S^{3}$ is called a tunnel number one link if there exists an arc $\tau$ properly embedded in $S^{3} \backslash n(L)$ such that $S^{3} \backslash n(L \cup \tau)$ is a handlebody. Then $\tau$ is called a tunnel for $L$.

Observe that the complement of a tunnel number one link has a genus-2 Heegaard splitting. Also, note that a tunnel one link has at most two components, and if it has two components, then any tunnel must have one endpoint on each component.

More generally, a knot is tunnel number $n$ if $n$ is the smallest number such that there exists a collection of $\operatorname{arcs}\left\{\tau_{1}, \ldots, \tau_{n}\right\}$ such that $S^{3} \backslash n\left(L \cup \tau_{1} \cup \cdots \cup \tau_{n}\right)$ is a handlebody.

\section{Fibered links.}

Definition 2.5. Let $L \subset S^{3}$ be a link. A Seifert surface for $L$ is a compact, orientable surface $F$ embedded in $S^{3}$ with no closed components such that $\partial F=L$.

Definition 2.6. A map $f: E \rightarrow B$ is a fibration with fiber $F$ if for every point $p \in B$, there is a neighborhood $U$ of $p$ and a homeomorphism $h: f^{-1}(U) \rightarrow U \times F$ such that $f=\pi_{1} \circ h$, where $\pi_{1}: U \times F \rightarrow U$ is projection to the first factor. The space $E$ is called the total space, and $B$ is called the base space. Each set $f^{-1}(b)$ is called a fiber, and is homeomorphic to $F$.

Definition 2.7. A link $L \subset S^{3}$ is said to be fibered if there is a fibration of $S^{3} \backslash n(L)$ over $S^{1}$, and the fibration is well-behaved near $L$. That is, each component $L_{i}$ of $L$ has a neighborhood $S^{1} \times D^{2}$, with $L_{i} \cong S^{1} \times\{0\}$ such that $\left.f\right|_{S^{1} \times\left(D^{2} \backslash\{0\}\right)}$ is given by $(x, y) \rightarrow y /|y|$.

Each fiber of a fibered link is a Seifert surface for the link. The complement of a fibered link is foliated by copies of this Seifert surface. Cutting along one of these Seifert surfaces produces a surface cross the interval.

Definition 2.8. Let $K$ be a fibered link in $S^{3}$. Then $S^{3} \backslash n(K)$ can be obtained from $F \times I$, with $F$ a fiber, by identification $(x, 0) \sim(h(x), 1)$, for $x \in F$, where $h: F \rightarrow F$ is an orientation-preserving homeomorphism which is the identity on $\partial F$. We call $h$ a monodromy map.

Theorems. Our starting point is the following theorem.

Theorem 2.9 [Scharlemann and Thompson 2003]. Suppose $K$ is a knot in $S^{3}$, and $\tau$ an unknotting tunnel for $K$. Then $\tau$ may be slid and isotoped until it is disjoint from some minimal-genus Seifert surface for $K$. 
The proof consists of arranging $K, \tau$, and a compressing disk for $S^{3} \backslash n(K \cup \tau)$ in some minimal fashion, and showing that if $K \cap \tau \neq \varnothing$, this would lead to a contradiction with those minimality assumptions. The result still holds for twocomponent fibered links.

Theorem 2.10. Suppose $K$ is an oriented, fibered link, and $\tau$ is an unknotting tunnel for $K$. Then $\tau$ may be slid and isotoped until it is disjoint from a fiber of $K$.

Our proof will largely mimic [Scharlemann and Thompson 2003].

Proof. By Theorem 2.9, if $K$ has just one component, then an unknotting tunnel can be isotoped and slid to be disjoint from a minimal-genus Seifert surface. But in a fibered knot complement, a fiber is the unique minimal-genus Seifert surface, so the result follows. Henceforth, let us assume that $K$ is a two-component link, and let the two components of $K$ be $K_{1}$ and $K_{2}$. Observe that $\tau$ has one endpoint on each of the components of $K$. Choose a fiber $F$, and slide and isotope $\tau$, so as to minimize the number of intersections between $\tau$ and $F$. Our goal will be to prove that $\tau \cap F=\varnothing$.

Suppose, to the contrary, that after the slides and isotopies above, $\tau \cap F$ is nonempty. Let $E$ be an essential disk in the handlebody $S^{3} \backslash n(K \cup \tau)$, chosen to minimize the number $|E \cap F|$ of components in $E \cap F$. If $|E \cap F|=0$, then the incompressible $F$ would lie in a solid torus, namely (a component of) $S^{3} \backslash n(K \cup$ $\tau \cup E$ ), and so be an annulus. The only fibered link with fiber an annulus is the Hopf link, in which case the result holds. So we may assume that $|E \cap F|>0$. Furthermore, since $F$ is incompressible, we may assume that $E \cap F$ consists entirely of arcs.

Let $e$ be an outermost arc of $E \cap F$ in $E$, cutting off a subdisk $E_{0}$ from $E$. If $e$ were inessential in $F \backslash \tau$, then we could surger $E$ along the trivial subdisk cut off by $e$. The result would be two disks, at least one of which is also essential in $S^{3} \backslash n(K \cup \tau)$, but with one fewer intersection with $F$, contradicting our assumption of minimality. Thus, the arc $e$ is essential in $F \backslash \tau$. Let $f=\partial\left(E_{0}\right) \backslash e$, an arc in $\partial n(K \cup \tau)$ with each end either on a longitude $\partial F \subset \partial n(K)$ or a meridian disk of $\tau$ corresponding to a point of $\tau \cap F$.

Now, either no meridian of $\tau$ is incident to an end of $f$, a meridian of $\tau$ is incident to exactly one end of $f$, or there is a meridian which is incident to both ends of $f$.

(1) If no meridian of $\tau$ is incident to an end of $f$, then both ends of $f$ lie on $\partial F \subset \partial n(K)$. If the interior of $f$ runs over $\tau$, we have finished, for $f$ is disjoint from $F$. Otherwise, the interior of $f$ lies entirely in $\partial n(K)$, and $e$ is either essential in $F$, or it is inessential.

(a) If $e$ is essential in $F$, then $E_{0}$ would be a boundary compression disk for $F$, contradicting the minimality of the genus of $F$. 
(b) If $e$ is inessential in $F$, then the disk $D_{0}$ that it cuts off from $F$ necessarily contains points of $\tau$ (since $e$ is essential in $F \backslash \tau$ ). But then we could replace $D_{0}$ by $E_{0}$, and the loop formed by $f$ and $\partial D_{0} \backslash e$ is either a trivial loop on one of the torus components of $\partial n(K)$, or it is an essential loop.

(i) If the loop formed by $f$ and $\partial D_{0} \backslash e$ is a trivial loop on the torus, say, $\partial n\left(K_{1}\right)$, then the new surface would, again, be a Seifert surface for $K$, consistent with the orientation of $K$ (and so be a fiber), but with fewer points of intersection $F \cap \tau$.

(ii) If the loop formed by $f$ and $\partial D_{0} \backslash e$ is essential in $\partial n\left(K_{1}\right)$, then the original disk $E_{0}$ could be slid across $D_{0}$ to show that $K_{1}$ is unknotted. But the interior of the disk $D_{0}$ is disjoint from $K_{2}$, so $K$ must be a split link, and split links do not fiber.

(2) If a meridian of $\tau$ is incident to exactly one end of $f$, then we can use $E_{0}$ to describe a simple isotopy of $\tau$ by sliding $\tau$ along $E_{0}$ which reduces the number of intersections between $\tau$ and $F$.

(3) If both ends of $f$ lie on the same meridian of $\tau$, then $e$ forms a loop in $F$, and the ends of $f$ adjacent to $e$ both run along the same subarc $\tau_{0}$ of $\tau$. Since $f$ is disjoint from $F, \tau_{0}$ terminates on, say, $\partial n\left(K_{1}\right)$.

Then since the interior of $f$ is disjoint from $F, f$ must intersect $\partial n\left(K_{1}\right)$ either in an inessential arc in the torus or in a longitudinal arc. That is, if $\tau_{0} \cap \partial n\left(K_{1}\right)$ were collapsed to a point $p$, then $f$ would either represent a trivial loop in $\pi_{1}\left(\partial n\left(K_{1}\right), p\right)$, or a nontrivial element. The former case is impossible, because the trivial disk cut off by $f$ cannot contain the other end of $\tau$ (since the other end of $\tau$ is on $\partial n\left(K_{2}\right)$ ). Thus, the disk could be isotoped away, reducing $|E \cap F|$. It follows that $f$ intersects the torus $\partial n\left(K_{1}\right)$ in a longitudinal arc. Then, $n\left(\tau_{0} \cup E_{0}\right)$ is a thickened annulus $A$, defining a parallelism in $S^{3}$ between $K_{1}$ and the loop $e$ on $F$. Now, the boundary component of $A$ on $\partial n\left(K_{1}\right)$ can be slid across $\partial n\left(K_{1}\right)$, away from $e$, onto $F$, parallel to $\partial F$ in $F$. Since $K$ is a fibered link, the image of $A$, call it $A^{\prime}$, is a product annulus in $S^{3} \backslash n(K \cup F) \cong F \times I$. But then this demonstrates that $e$ itself is parallel to $\partial F$ in $F$. Then, substituting $A$ for the annulus between $e$ and $\partial F$ in $F$ would create a Seifert surface of the same genus, still consistent with the orientation of $K$, and thus a fiber, but with fewer intersections with $\tau$.

In all cases, we obtain contradictions, and conclude that $\tau$ and $F$ can be arranged to be disjoint.

Another theorem that we will find useful is also given by Scharlemann and Thompson. Ni [2009] proves a more general result, though we will not need it here. 
Theorem 2.11 [Scharlemann and Thompson 2009]. Suppose $F$ is a compact orientable surface, $L$ is a knot in $F \times I$, and $(F \times I)_{\text {surg }}$ is the 3-manifold obtained by some nontrivial surgery on $L$. If $F \times\{0\}$ compresses in $(F \times I)_{\text {surg }}$, then $L$ is parallel to an essential simple closed curve in $F \times\{0\}$. Moreover, the annulus that describes the parallelism determines the slope of the surgery.

The proof relies on sutured manifold theory, and a theorem of Gabai [1989]. Gabai proves the result for an annulus cross the interval. The idea of Scharlemann and Thompson's proof is to find product disks or annuli in $(F \times I)$ disjoint from the knot, and cut along these product pieces to reduce the complexity of the surface in question. This, with some additional work, allows them to apply the results of Gabai.

\section{Pushing a tunnel into a fiber}

Proof of Theorem 1.1. By Theorem 2.10, $\tau$ can be isotoped and slid to be disjoint from a fiber. Let $F=F^{\prime} \backslash n(K)$. Cut $S^{3} \backslash n(K)$ along $F$, to obtain $N \cong F \times I$, a handlebody. Then $\tau \subset N$.

Now, as $\tau$ is an unknotting tunnel, there exists a compressing disk for $\partial n(K \cup \tau)$ in $S^{3} \backslash n(K \cup \tau)$, say $D^{\prime}$. Note that $D^{\prime} \cap F \neq \varnothing$, for otherwise $F$ would be an essential surface in the solid torus $\left(S^{3} \backslash n(K \cup \tau)\right) \mid D^{\prime}$, and thus a disk.

Consider $D^{\prime} \cap F$. Since $F$ is incompressible and $N$ is irreducible, by standard innermost disk arguments we may assume there are no simple closed curves of intersection. Let $\alpha$ be an arc of intersection which is outermost in $D^{\prime}$, cutting off a subdisk $D$. Then, $D$ is a disk in $N$ with boundary consisting of three types of arcs: a single essential arc in $F=F \times\{0\}, \alpha$; (several) arcs in $\partial n(K)$, call them $\nu_{i}$; and (several) arcs in $\partial n(\tau), \lambda_{j}$. We may assume that every $\operatorname{arc}$ of $D \cap \partial n(\tau)$ is an essential spanning arc of the annulus $\partial n(\tau)$, for trivial arcs can be removed by isotopy.

Now, consider the double of $N$, along the vertical boundary $\partial F \times I$. In other words, let $\widehat{N}$ be the result of gluing two copies of $N$ together by the identity along $\partial F \times I$. Similarly, let $\widehat{\tau}$ be the result of gluing two copies of $\tau$, one in each copy of $N$, along the boundary points; let $\widehat{D}$ come from two copies of $D$, one in each copy of $N$, glued along the $v_{i}$; and let $\widehat{\alpha}$ come from two copies of $\alpha$ in the same way.

Then $\widehat{D}$ is a planar surface with one boundary component corresponding to $\widehat{\alpha}$, and several components coming from $\widehat{\lambda}_{j}$, the doubles of $\lambda_{j}$ (see Figure 1).

Then, $\bigcup_{j} \widehat{\lambda}_{j}$ is a collection of (parallel) simple closed curves on the torus $\partial n(\widehat{\tau})$. Call the slope determined by these curves $\lambda$. If we perform $\lambda$-surgery on $\widehat{\tau}$, the result is to cap off $\widehat{D}$ with disks. Since $\alpha$ was essential in $F$, $\widehat{\alpha}$ is essential in $\widehat{F}$, so our capped-off surface is a compression disk for $\widehat{F}$ in $\widehat{N} \cong \widehat{F} \times I$. 


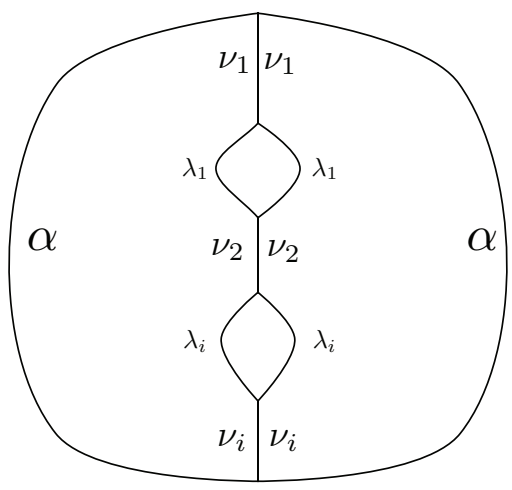

Figure 1. $\widehat{D}$.

By Theorem 2.11, $\widehat{\tau}$ is parallel to an essential closed curve in $\widehat{F} \times\{0\}$. That is, there exists an annulus $A$ properly embedded in $\widehat{F} \times I$ with one boundary component on $\widehat{F} \times\{0\}$, say $\psi$, and the other boundary component on $\partial n(\widehat{\tau})$, parallel to $\widehat{\tau}$, say $\phi$.

Since $\phi$ is parallel to $\widehat{\tau}$, it must be a longitude of $\partial n(\widehat{\tau})$, and in particular, $|\phi \cap(\partial F \times I)|=2$. So there are only two possibilities for arcs of intersection between $A$ and $\partial F \times I$ incident to $\phi$. Either there is one arc of intersection which is trivial in $A$, or there are two arcs of intersection, both of which are essential in $A$ (see Figure 2). The former case is impossible, because then the subdisk of $A$ cut off by the arc would show that $\tau$ was parallel into $\partial n(K)$, which would imply that $K$ was trivial. Therefore, there are exactly two arcs of $A \cap(\partial F \times I)$, both of which are essential in $A$.

If there were trivial arcs incident to $\psi$, then an outermost such arc in $A$ would give rise to a boundary compression for $F \times\{0\}$ in $S^{3} \backslash n(K)$. This is impossible as well, so $\partial F \times I$ intersects $A$ in precisely two essential arcs, with no trivial arcs. Cutting $A$ along these arcs provides a parallelism between $\tau$ and the arc $\psi \cap(F \times\{0\}) \subset \widehat{F} \times\{0\}$. Thus, $\tau$ can be isotoped to lie in the fiber.
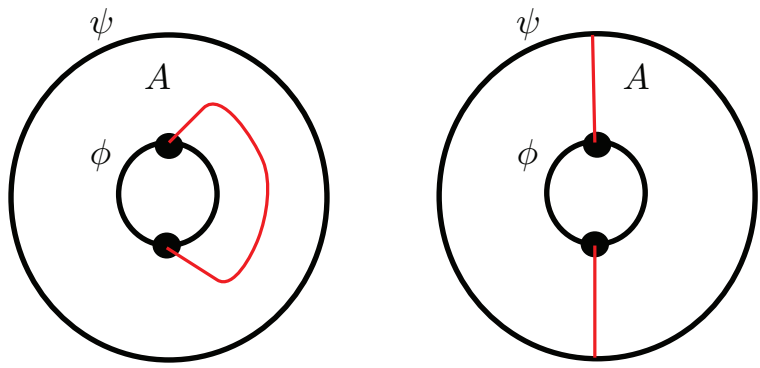

Figure 2. Arcs of $A \cap \partial F \times I$ incident to $\phi$. 


\section{References}

[Adams 1995] C. C. Adams, "Unknotting tunnels in hyperbolic 3-manifolds", Math. Ann. 302:1 (1995), 177-195. MR 96c:57004 Zbl 0830.57009

[Adams and Reid 1996] C. C. Adams and A. W. Reid, "Unknotting tunnels in two-bridge knot and link complements”, Comment. Math. Helv. 71:4 (1996), 617-627. MR 98h:57009 Zbl 0873.57006

[Akiyoshi et al. 1997] H. Akiyoshi, Y. Nakagawa, and M. Sakuma, "Shortest vertical geodesics of manifolds obtained by hyperbolic Dehn surgery on the Whitehead link", pp. 433-448 in KNOTS (Tokyo, 1996), edited by S. Suzuki, World Scientific, River Edge, NJ, 1997. MR 99k:57034 Zbl 0960.57013

[Baker et al. 2008] K. L. Baker, J. E. Grigsby, and M. Hedden, "Grid diagrams for lens spaces and combinatorial knot Floer homology", Int. Math. Res. Not. 2008:10 (2008), Art. ID rnm024. MR 2009h:57012 Zbl 1168.57009

[Boileau et al. 1988] M. Boileau, M. Rost, and H. Zieschang, "On Heegaard decompositions of torus knot exteriors and related Seifert fibre spaces", Math. Ann. 279:3 (1988), 553-581. MR 89a:57013 Zbl 0616.57008

[Cooper et al. 2010] D. Cooper, M. Lackenby, and J. S. Purcell, "The length of unknotting tunnels", Algebr. Geom. Topol. 10:2 (2010), 637-661. MR 2011c:57014 Zbl 1194.57021

[Eudave Muñoz and Uchida 1996] M. Eudave Muñoz and Y. Uchida, "Non-simple links with tunnel number one”, Proc. Amer. Math. Soc. 124:5 (1996), 1567-1575. MR 96g:57007 Zbl 0858.57008

[Gabai 1989] D. Gabai, "Surgery on knots in solid tori”, Topology 28:1 (1989), 1-6. MR 90h:57005 Zbl 0678.57004

[Giroux 2002] E. Giroux, "Géométrie de contact: de la dimension trois vers les dimensions supérieures", pp. 405-414 in Proceedings of the International Congress of Mathematicians (Beijing, 2002), vol. 2, edited by T. T. Li et al., Higher Ed. Press, Beijing, 2002. MR 2004c:53144 Zbl 1015.53049 arXiv math/0305129

[Goda et al. 2000] H. Goda, M. Scharlemann, and A. Thompson, "Levelling an unknotting tunnel", Geom. Topol. 4 (2000), 243-275. MR 2002h:57011 Zbl 0958.57007

[Heath and Song 2005] D. J. Heath and H.-J. Song, "Unknotting tunnels for P(-2, 3, 7)", J. Knot Theory Ramifications 14:8 (2005), 1077-1085. MR 2007e:57008 Zbl 1115.57003

[Hedden 2007] M. Hedden, "Knot Floer homology of Whitehead doubles", Geom. Topol. 11 (2007), 2277-2338. MR 2008m:57030 Zbl 1187.57015

[Johnson 2008] J. Johnson, "Surface bundles with genus two Heegaard splittings", J. Topol. 1:3 (2008), 671-692. MR 2009f:57025 Zbl 1148.57029

[Jones 1995] A. C. Jones, "Composite two-generator links", Topology Appl. 67:3 (1995), 165-178. MR 97f:57008 Zbl 0854.57005

[Kobayashi 1999] T. Kobayashi, "Classification of unknotting tunnels for two bridge knots", pp. 259-290 in Proceedings of the Kirbyfest (Berkeley, CA, 1998), edited by J. Hass and M. Scharlemann, Geom. Topol. Monogr. 2, Geom. Topol. Publ., Coventry, 1999. MR 2000j:57013 Zbl 0962. 57003

[Lackenby 2005] M. Lackenby, "Classification of alternating knots with tunnel number one", Comm. Anal. Geom. 13:1 (2005), 151-185. MR 2006e:57007 Zbl 1084.57007

[Morimoto 1994] K. Morimoto, "On composite tunnel number one links", Topology Appl. 59:1 (1994), 59-71. MR 95f:57016 Zbl 0821.57006

[Morimoto and Sakuma 1991] K. Morimoto and M. Sakuma, "On unknotting tunnels for knots", Math. Ann. 289:1 (1991), 143-167. MR 92e:57015 Zbl 0697.57002 
[Ni 2007] Y. Ni, "Knot Floer homology detects fibred knots", Invent. Math. 170:3 (2007), 577-608. MR 2008j:57053 Zbl 1138.57031

[Ni 2009] Y. Ni, "Dehn surgeries that yield fibred 3-manifolds", Math. Ann. 344:4 (2009), 863-876. MR 2010m:57028 Zbl 1227.57012

[Ozsváth and Szabó 2005] P. Ozsváth and Z. Szabó, "On knot Floer homology and lens space surgeries”, Topology 44:6 (2005), 1281-1300. MR 2006f:57034 Zbl 1077.57012

[Saito 2007] T. Saito, "Dehn surgery and (1, 1)-knots in lens spaces", Topology Appl. 154:7 (2007), 1502-1515. MR 2008f:57011 Zbl 1115.57005

[Sakuma 1996] M. Sakuma, "Unknotting tunnels and canonical decompositions of punctured torus bundles over a circle”, Sūrikaisekikenkyūsho Kōkyūroku 967 (1996), 58-70. MR 98k:57021 Zbl 0924.57020

[Sakuma and Weeks 1995] M. Sakuma and J. Weeks, "Examples of canonical decompositions of hyperbolic link complements", Japan. J. Math. (N.S.) 21:2 (1995), 393-439. MR 96j:57013 Zbl 0858.57021

[Scharlemann and Thompson 2003] M. Scharlemann and A. Thompson, "Unknotting tunnels and Seifert surfaces", Proc. London Math. Soc. (3) 87:2 (2003), 523-544. MR 2004e:57015 Zbl 1047. 57008

[Scharlemann and Thompson 2009] M. Scharlemann and A. A. Thompson, "Surgery on a knot in (surface × I)", Algebr. Geom. Topol. 9:3 (2009), 1825-1835. MR 2010m:57018 Zbl 1197.57011

[Williams 2007] M. J. Williams, "Lens space surgeries \& primitive/Seifert type constructions", preprint, 2007. arXiv 0712.1797

Received March 17, 2012. Revised June 4, 2012.

MatT RathBun

DEPARTMENT OF MATHEMATICS

HUXLEY BUILDING 6M33

IMPERIAL COLLEGE LONDON, SOUTH KENSINGTON CAMPUS

LONDON, SW7 2AZ

UNITED KINGDOM

m.rathbun@imperial.ac.uk

http://www2.imperial.ac.uk/ mrathbun/ 


\title{
PACIFIC JOURNAL OF MATHEMATICS
}

\author{
http://pacificmath.org \\ Founded in 1951 by \\ E. F. Beckenbach (1906-1982) and F. Wolf (1904-1989)
}

\section{EDITORS}

V. S. Varadarajan (Managing Editor)

Department of Mathematics

University of California

Los Angeles, CA 90095-1555

pacific@math.ucla.edu

Vyjayanthi Chari

Department of Mathematics

University of California

Riverside, CA 92521-0135

chari@math.ucr.edu

\section{Robert Finn}

Department of Mathematics Stanford University

Stanford, CA 94305-2125

finn@math.stanford.edu

Kefeng Liu

Department of Mathematics

University of California

Los Angeles, CA 90095-1555

liu@math.ucla.edu
Darren Long

Department of Mathematics

University of California

Santa Barbara, CA 93106-3080

long@math.ucsb.edu

Jiang-Hua Lu

Department of Mathematics

The University of Hong Kong

Pokfulam Rd., Hong Kong jhlu@maths.hku.hk

Alexander Merkurjev

Department of Mathematics

University of California

Los Angeles, CA 90095-1555

merkurev@math.ucla.edu
Sorin Popa

Department of Mathematics University of California

Los Angeles, CA 90095-1555 popa@math.ucla.edu

Jie Qing

Department of Mathematics

University of California

Santa Cruz, CA 95064

qing@cats.ucsc.edu

Jonathan Rogawski

Department of Mathematics

University of California

Los Angeles, CA 90095-1555

jonr@math.ucla.edu

\section{PRODUCTION}

pacific@math.berkeley.edu

\section{SUPPORTING INSTITUTIONS}

ACADEMIA SINICA, TAIPEI

CALIFORNIA INST. OF TECHNOLOGY INST. DE MATEMÁTICA PURA E APLICADA KEIO UNIVERSITY

MATH. SCIENCES RESEARCH INSTITUTE NEW MEXICO STATE UNIV.

OREGON STATE UNIV.

\author{
STANFORD UNIVERSITY \\ UNIV. OF BRITISH COLUMBIA \\ UNIV. OF CALIFORNIA, BERKELEY \\ UNIV. OF CALIFORNIA, DAVIS \\ UNIV. OF CALIFORNIA, LOS ANGELES \\ UNIV. OF CALIFORNIA, RIVERSIDE \\ UNIV. OF CALIFORNIA, SAN DIEGO \\ UNIV. OF CALIF., SANTA BARBARA
}

\author{
UNIV. OF CALIF., SANTA CRUZ \\ UNIV. OF MONTANA \\ UNIV. OF OREGON \\ UNIV. OF SOUTHERN CALIFORNIA \\ UNIV. OF UTAH \\ UNIV. OF WASHINGTON \\ WASHINGTON STATE UNIVERSITY
}

These supporting institutions contribute to the cost of publication of this Journal, but they are not owners or publishers and have no responsibility for its contents or policies.

See inside back cover or pacificmath.org for submission instructions.

The subscription price for 2012 is US \$420/year for the electronic version, and \$485/year for print and electronic.

Subscriptions, requests for back issues from the last three years and changes of subscribers address should be sent to Pacific Journal of Mathematics, P.O. Box 4163, Berkeley, CA 94704-0163, U.S.A. Prior back issues are obtainable from Periodicals Service Company, 11 Main Street, Germantown, NY 12526-5635. The Pacific Journal of Mathematics is indexed by Mathematical Reviews, Zentralblatt MATH, PASCAL CNRS Index, Referativnyi Zhurnal, Current Mathematical Publications and the Science Citation Index.

The Pacific Journal of Mathematics (ISSN 0030-8730) at the University of California, c/o Department of Mathematics, 969 Evans Hall, Berkeley, CA 94720-3840, is published monthly except July and August. Periodical rate postage paid at Berkeley, CA 94704, and additional mailing offices. POSTMASTER: send address changes to Pacific Journal of Mathematics, P.O. Box 4163, Berkeley, CA 94704-0163.

PJM peer review and production are managed by EditFLOW ${ }^{\mathrm{TM}}$ from Mathematical Sciences Publishers.

PUBLISHED BY PACIFIC JOURNAL OF MATHEMATICS

at the University of California, Berkeley 94720-3840

A NON-PROFIT CORPORATION

Typeset in LATEX

Copyright $(02012$ by Pacific Journal of Mathematics 


\section{PACIFIC JOURNAL OF MATHEMATICS}

Volume $259 \quad$ No. $2 \quad$ October 2012

Flag subdivisions and $\gamma$-vectors

257

Christos A. ATHANASIAdis

Rays and souls in von Mangoldt planes

279

IGOR BELEGRADEK, ERIC CHOI and NOBUHIRO INNAMI

Isoperimetric surfaces with boundary, II

ABRAHAM FRANDSEN, DONALD SAMPSON and NEIL

STEINBURG

Cyclic branched coverings of knots and quandle homology

YUICHI KABAYA

On a class of semihereditary crossed-product orders

JOHN S. KAUTA

An explicit formula for spherical curves with constant torsion

DEMETRE KAZARAS and IVAN STERLING

Comparing seminorms on homology

JEAN-FranÇOIS LAFONT and CHRISTOPHE PITTET

Relatively maximum volume rigidity in Alexandrov geometry

NAN LI and XIAOCHUN RONG

Properness, Cauchy indivisibility and the Weil completion of a group of 421 isometries

Antonios Manoussos and Polychronis Strantzalos

Theta lifts of strongly positive discrete series: the case of $(\tilde{\mathrm{Sp}}(n), O(V)) 445$

IVAN MATIĆ

Tunnel one, fibered links

MATt RATHBUN

Fusion symmetric spaces and subfactors

HANS WENZL 